\title{
Case Series of Chordoma in Three Different Locations: Clival, Cervical Spine, and Sacrococcygeal
}

\author{
Irfan Deny Sanjaya ${ }^{\text {a*}}$, Sri Andreani Utomo ${ }^{\mathrm{a}}$ \\ a irfan.deny.sanjaya-2017@fk.unair.ac.id
}

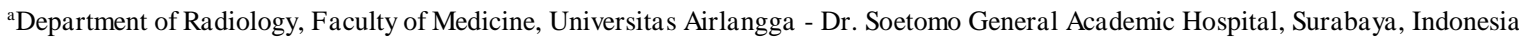

\begin{abstract}
Background: Chordoma is a relatively rare malignant, slow growing, and locally aggressive tumour that arise from embryonic remnants of the primitive notochord. It accounts for $4 \%$ of all primary bone tumours. Chordomas can present a diagnostic challenge due to the rare occurrence and a tendency to involve any region within the craniospinal axis.

Case Presentation: First case, 25-y-o female with cephalgia. CT scan shows lytic clival bone destruction. MRI show lobulated isointense mass on T1-WI, hyperintense on T2-WI and heterogenous contrast-enhancement. The mass extending to the many structures, destructing the Atlas, infiltrating perivertebral and epidural space. The patient received endoscopic tumour excision and confirmed as chordoma. Second case, 50-y-o male with tetraparesis, urinary and bowel incontinence. CT scan shows destruction of the first and second cervical spine. MRI shows the same characteristics as the first case. The mass compressing spinal cord, infiltrating the right perivertebral and orophrayngeal space. The patient received laminectomy and confirmed as chordoma. Third case, 50-y-o male with large soft tissue bulging at gluteal region. CT scan 3D surface rendering shows destruction of the first sacral spine until coccygeal. MRI shows the same characteristics as the first and second case. The mass infiltrating gluteal and piriform muscles, encasing left internal iliac artery. The mass further pathologically confirmed as chordoma.

Conclusion: Patients might experience different symptoms. Imaging studies classically show a destructive bone mass with lobular growth, high water content (high T2 signal intensity), foci of haemorrhage and calcification. Differential diagnosis are also depend on the location. Physaliferous cells is the hallmark of chordoma. Surgical intervention is the recommended treatment modality.
\end{abstract}

Keywords: Chordoma; Clival; Cervical; Sacrococcygeal

\section{Background}

Chordoma is a relatively rare malignant, slow growing, and locally aggressive tumour that arise from embryonic remnants of the primitive notochord. Remnants of the notochord are usually within bone, remain in or close to the midline. It accounts for $4 \%$ of all primary bone tumours (Baratti, 2003; Shors SM et al., 2006; Kyriakos M et al., 2011; Gerber S et al., 2008). The three most common sites for a chordoma are the 
sacrococcygeal area, the sphenooccipital area, and the C2 vertebra (Muro $\mathrm{K}$ et al., 2007). Chordomas can present a diagnostic challenge due to the rare occurrence and a tendency to involve any region within the craniospinal axis. We attempted to present the clinical, imaging, and pathologic features of chordoma in three different locations. Three pathologically confirmed cases of chordoma in different locations which are clival, cervical spinal, and sacrococcygeal were presented with different clinical symptoms and several radiologic examinations.

\section{Case Presentation}

First case, 25-year-old female presented with cephalgia for past 7 months. She also experienced diplopia, right third-fourth-sixth and left sixth cranial nerve palsy. See Figure 1, Axial Computed Tomography (CT) shows lytic clival bone destruction replaced by soft tissue mass compressing pons to the posterior (a). Sagittal TI-WI (b) and Sagittal contrast-enhanced TI-WI (c) Magnetic Resonance Imaging (MRI) show isointense mass with multiple hyperintense foci and heterogenous contrast-enhancement, size about $7.7 \times 6.4 \times 5.5 \mathrm{~cm}$.
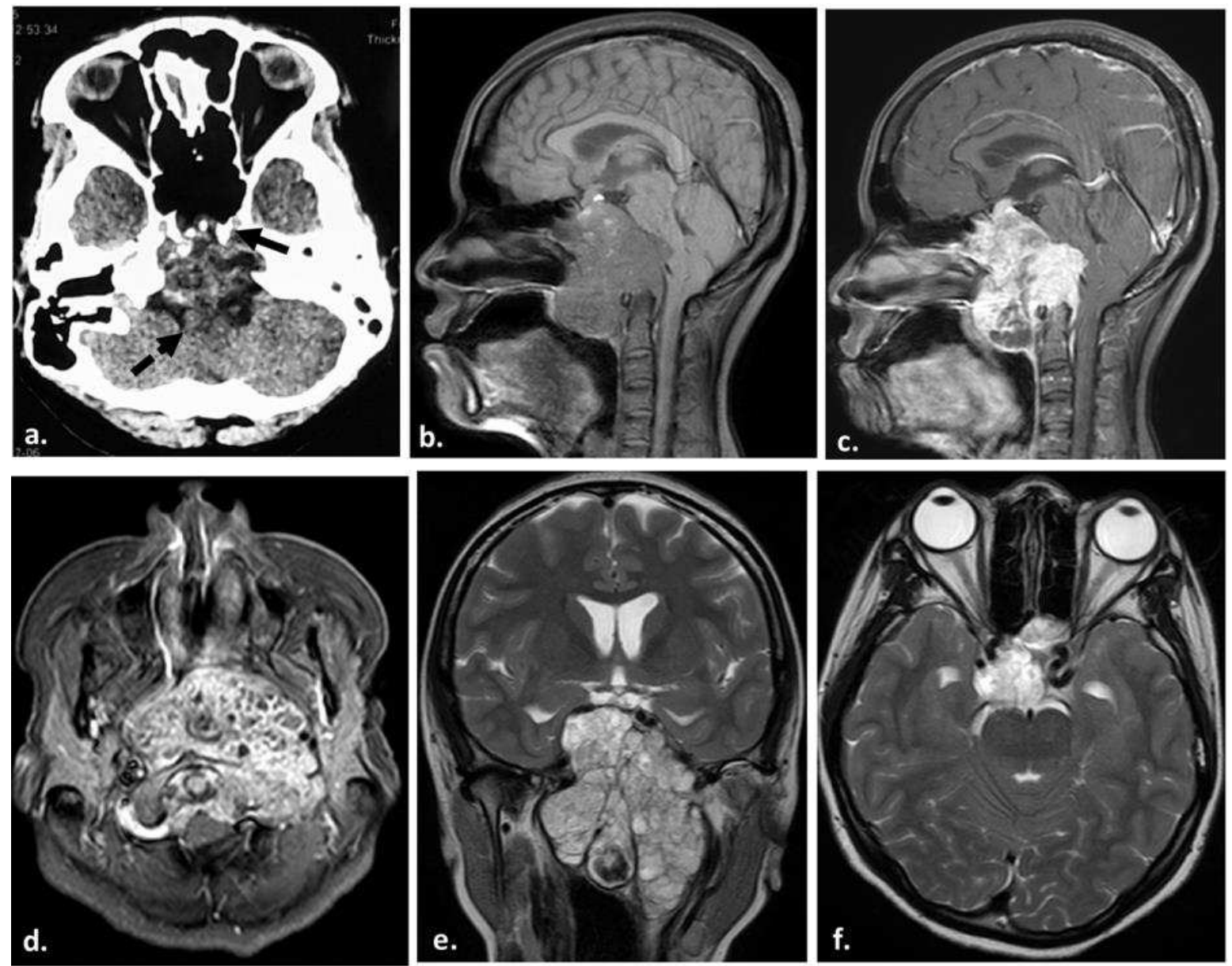

Figure 1. Clival Chordoma 
The mass extended anteriorly to the sphenoid sinus and nasopharynx; posteriorly compressing midbrain, pons, medulla oblongata, and cerebellum; inferiorly compressing the palate. Axial contrast-enhanced TI-WI MRI (d), the mass displacing both internal carotid arteries; destructing left anterior and posterior arch of Atlas, infiltrating perivertebral and epidural space; and displacing parapharyngeal space. Coronal (e) and Axial T2-WI (f) show lobulated hyperintense mass which extend superiorly compressing cavernous sinus (right more dominant) and left optic nerve. Then, the patient received endoscopic tumour excision (g,h) and pathologically confirmed as chordoma, epitheloid cells with eosinophilic to clear, bubbly cytoplasm arranged in nest, chords and individual cell in a myxoid stroma (i).

Second case, 50-year-old male presented with hypoesthesia in all four limbs for 2 years, tetraparesis for 1 month, also urinary and bowel incontinence. See Figure 2, CT scan (a, b, and c) shows destruction of the first and second cervical spine, replaced by isodense soft tissue mass. Sagittal MRI shows the characteristics of the mass which isointense on T1-WI (d), heterogenous contrast-enhancement on contrast enhanced T1-WI (e), hyperintense on T2-WI (f). MR myelography (g) shows partial obstruction of the cerebrospinal fluid flow.
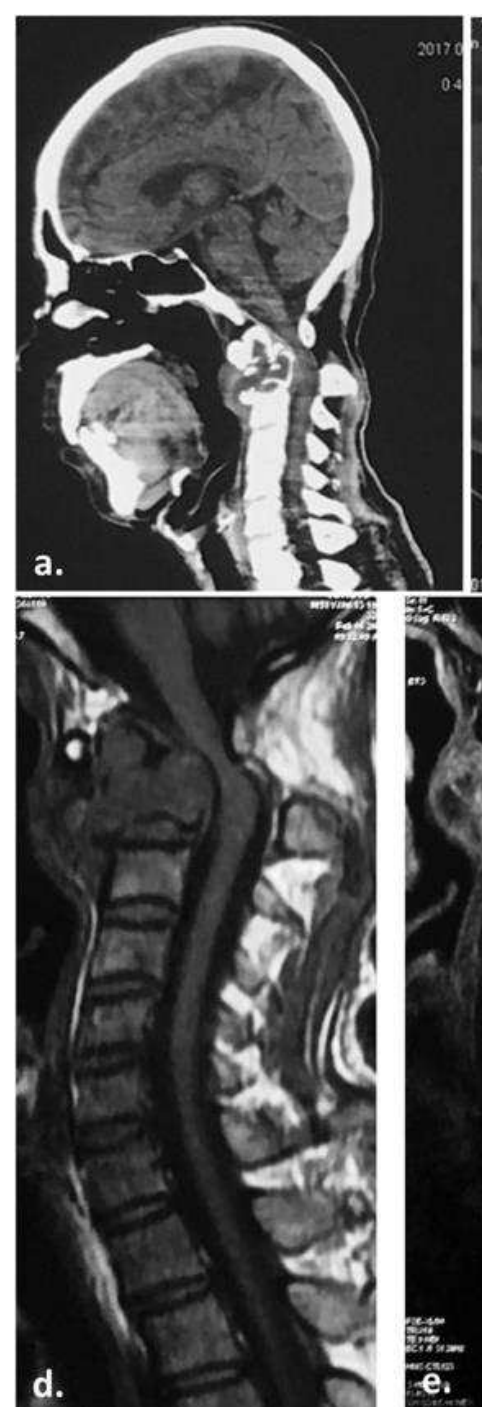
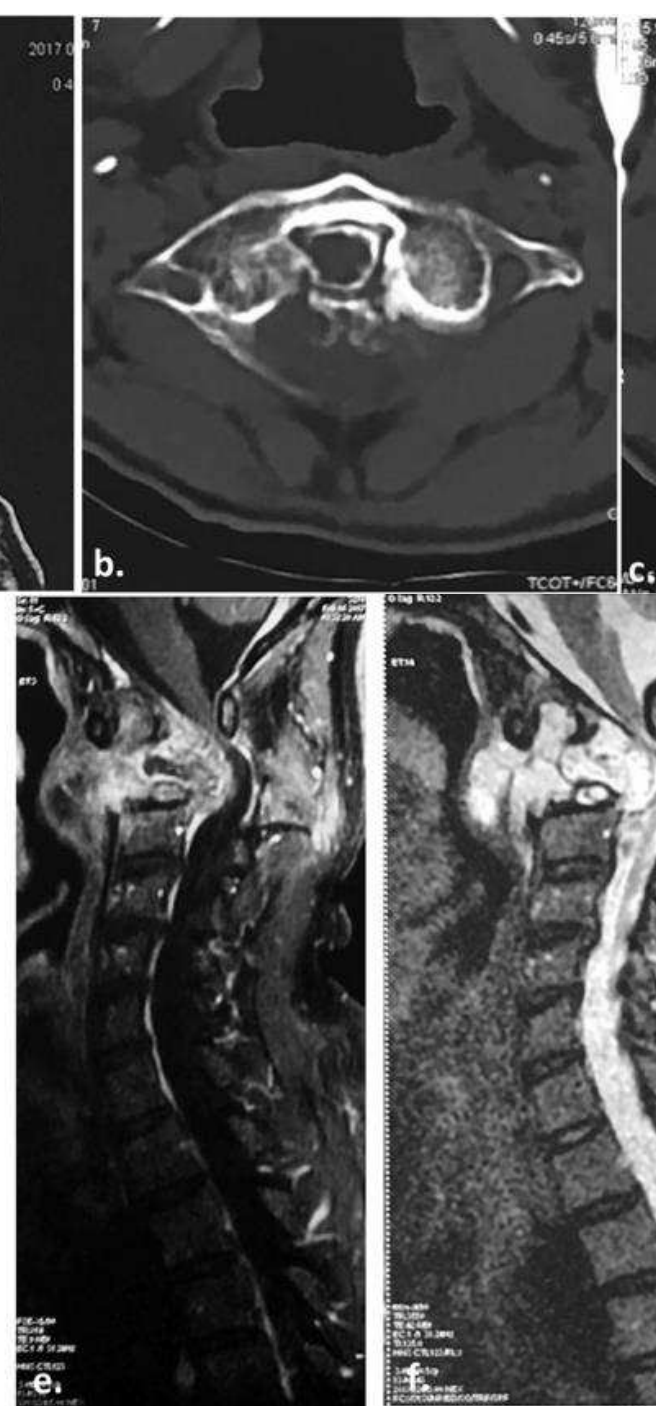
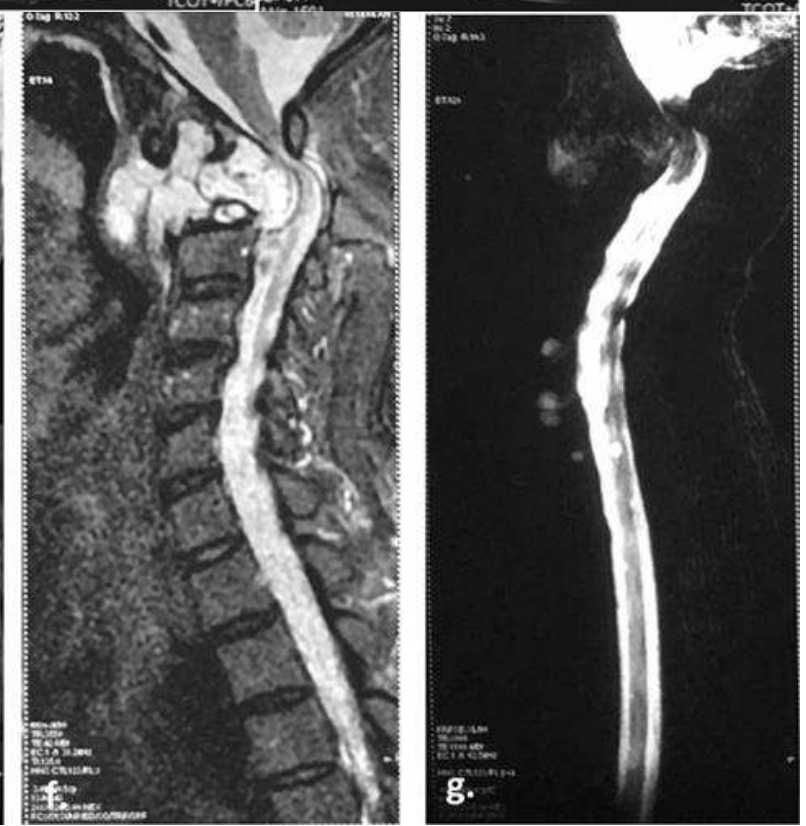


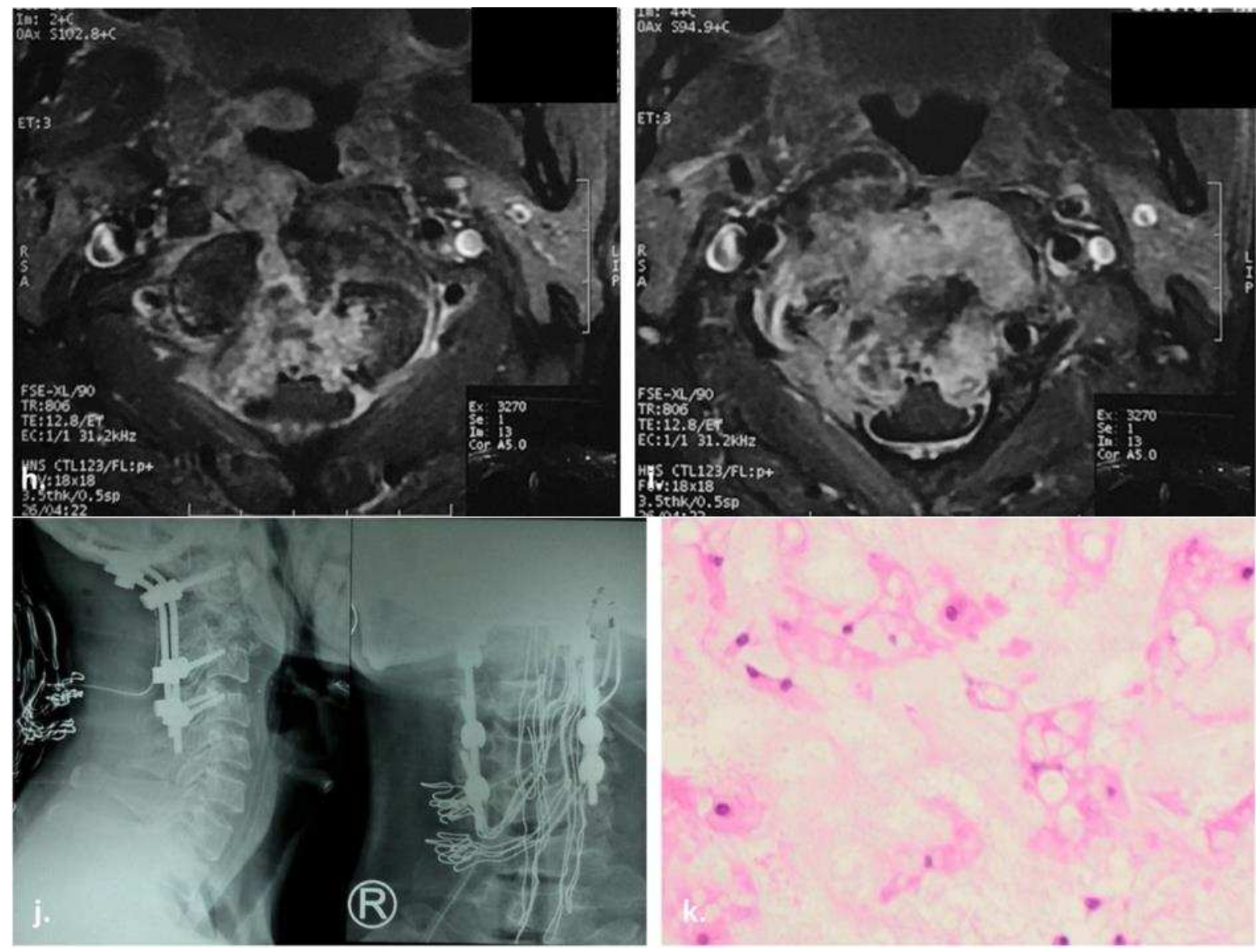

Figure 2. Cervical Spine Chordoma

Axial contrast enhanced T1-WI MRI (h, i) demonstrates the extension of the mass which is compressing spinal cord at those level, encasing right and displacing left vertebral artery, infiltrating to the right perivertebral space and parapharyngeal muscle with oropharyngeal extension. Then, the patient received first and second cervical spine laminectomy ( $\mathrm{j}$ ) and pathology approved abundance of physaliferous cells as the hallmark of chordoma $(\mathrm{k})$.

Third case, 50-year-old male presented with large soft tissue bulging at gluteal region, sacral pain that worsened when sitting and constipation over the past several months. He reported no history of bowel or bladder incontinence, motor weakness, or sensory paresthesias. See Figure 3, The patient complained about large soft tissue bulging at gluteal region (a). Ultrasound (b) demonstrates lobulated soft tissue mass at gluteal region with sacral destruction. Lumbosacral x-ray (c), Pelvis x-ray (d), and Pelvis CT scan 3D surface rendering (e) show destruction of the first sacral spine until coccygeus, extend to the both sacroiliac joint, both iliac bone, and left ischium bone. Sagittal T1-WI MRI (f) shows large isointense mass with several hyperintense areas. The mass appear lobulated and hyperintense on Sagittal T2-WI MRI (g), compressing bladder and rectum to the anterior with circumscribed margin, also displacing conus medullaris to the superoposterior. Axial T2-WI MRI (h) shows the mass infiltrating gluteal and piriform muscles, displacing right and encasing left internal iliac arteries. The cells have small round nuclei and abundant vacuolated cytoplasm (physaliferou), so pathologically confirmed as chordoma. 


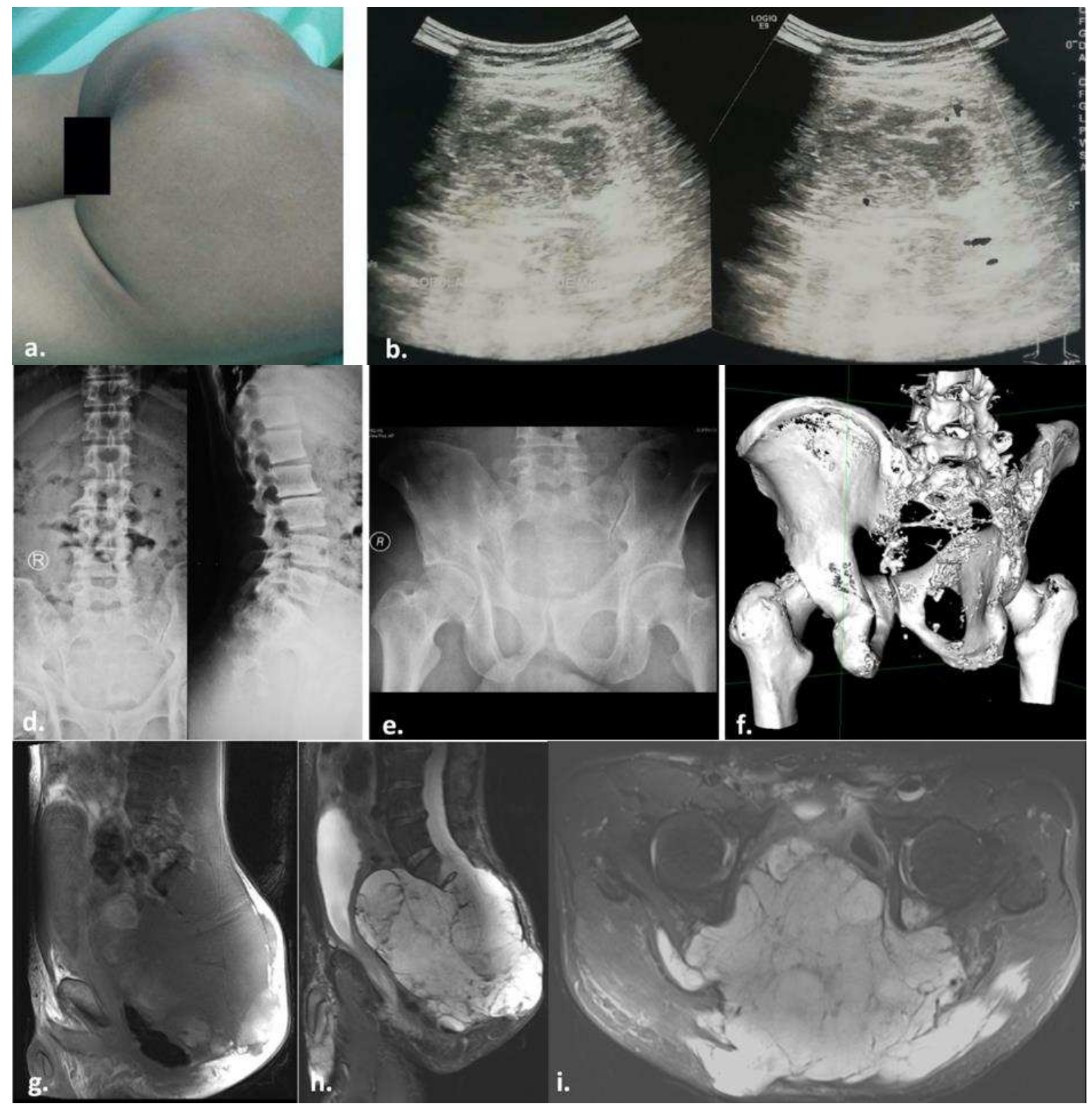

Figure 3. Sacrococcygeal Chordoma

\section{Conclusion}

Patients with chordoma might experience different symptoms depend on its location and extension. Imaging studies classically show a destructive bone mass with lobular growth, high water content (high T2 signal intensity), foci of haemorrhage and calcification. Differential diagnosis are also depend on the location. Physaliferous cells is the pathologic hallmark of chordoma and surgical intervention is the recommended treatment modality. 


\section{Acknowledgements}

I would like to dedicate my gratitudes to all lecturers of Departement of Radiology, Faculty of Medicine, Universitas Airlangga - Dr. Soetomo General Academic Hospital, Surabaya, Indonesia

\section{References}

Baratti D. Chordoma: natural history and results in 28 patients treated at a single institution. Ann Surg Oncol. 2003;10(3):291-296.

Shors SM, et al. Best cases from the AFIP: myxopapillary ependymoma of the sacrum. RadioGraphics 2006;26 (Spec Issue):S111-S1163.

Kyriakos M. Benign notochordal lesions of the axial skeleton: a review and current appraisal. Skelet Radiol. 2011;40(9):1141-52.

Gerber S, Ollivier L, Leclère J, et al. Imaging of sacral tumours. Skeletal Radiol 2008;37(4):277-289.

Muro K, Das S, Raizer JJ. Chordomas of the craniospinal axis: multimodality surgical, radiation and medical management strategies. Expert Rev Neurother 2007;7(10):1295-1312. 